\title{
基于超分子晶体制备超细铂纳米颗粒用于催化加氢硝基苯
}

\author{
张晓萌 ${ }^{a}$ 李希雅 ${ }^{a}$ 熊晚枫 ${ }^{b}$ 李红芳*,,$b$ 曹荣 ${ }^{a, b}$ \\ ( ${ }^{a}$ 福州大学 化学学院 福州 350108) \\ $\left({ }^{b}\right.$ 中国科学院福建物质结构研究所 结构化学国家重点实验室 福州 350002)
}

\begin{abstract}
摘要 由于纳米催化剂的独特催化活性，因而开发稳定的超细纳米催化剂的制备方法引起了广大科研工作者们的关 注. 然而, 复杂的合成过程和结构的不稳定性已经成为纳米催化剂研究和实际应用的瓶颈. 本工作通过原位还原氯铂 酸和十甲基五元瓜环 $\left(\mathrm{Me}_{10} \mathrm{CB}[5]\right)$ 构筑的超分子晶体材料, 成功制备了粒径尺寸约为 $3.0 \mathrm{~nm}$ 的超细铂纳米粒子. $\mathrm{Me}_{10} \mathrm{CB}[5]$ 的空间限域效应成功抑制铂纳米颗粒的团聚; 瓜环端口羰基氧与铂纳米粒子表面的相互作用防止铂纳米粒 子脱落, 确保催化剂的高稳定性. 该铂纳米催化剂对于温和条件下催化加氢硝基苯反应具有优良的催化活性、高稳定 性和高化学选择性. 这项工作为纳米催化剂的制备开辟了一条新途径, 为纳米催化剂在电化学、能源科学和环境保护 等许多重要领域的应用提供了基础.

关键词＼cjkstart超分子晶体; Pt 纳米颗粒; 硝基芳烃; 催化加氢
\end{abstract}

\section{Ultrafine Platinum Nanoparticles Derived from Supramolecular Crystal for Catalytic Hydrogenation of Nitroarenes}

\author{
Xiaomeng Zhang ${ }^{a} \quad \mathrm{Xiya} \mathrm{Li}^{a} \quad$ Wanfeng Xiong $^{b} \quad$ Hongfang $\mathrm{Li}^{*}, a, b \quad \mathrm{Rong} \mathrm{Cao}^{a, b}$ \\ $\left({ }^{a}\right.$ College of Chemistry, Fuzhou University, Fuzhou 350108, China $)$ \\ $\left({ }^{b}\right.$ State Key Laboratory of Structural Chemistry, Fujian Institute of Research on the Structure of Matter, \\ Chinese Academy of Sciences, Fuzhou 350002, China)
}

\begin{abstract}
The development of a general synthetic approach for stable ultrafine heterogeneous nanocatalysts has attracted extensive attentions worldwide. However, the complex synthetic process and structure unstability have been recognized as the bottleneck of their investigation and practical application. Herein, a convenient and high selective platinum nanocatalyst was developed based from crystalline supramolecular hybrid solid materials. The macrocyclic decamethylcucurbit[5]uril $\left(\mathrm{Me}_{10} \mathrm{CB}[5]\right)$ and $\left[\mathrm{PtCl}_{6}\right]^{2-}$ anions were firstly self-assembled into cyrstalline supramolecular solid in the presence of different alkali metal ions $\left(\mathrm{Li}^{+}, \mathrm{Na}^{+}, \mathrm{K}^{+}\right)$. Then, ultrafine platinum (Pt) nanoparticles (NPs) have been successfully synthesized through in-situ reduction of cyrstalline supramolecular assemblies under mild thermal treatment at $\mathrm{H}_{2}$ atmosphere. Uniform Pt NPs $(c a .3 .0 \mathrm{~nm})$ are produced and deposited on $\mathrm{Me}_{10} \mathrm{CB}[5]$ substrate to form $\mathrm{M}-\mathrm{Pt} @ \mathrm{Me}_{10} \mathrm{CB}[5]\left(\mathrm{M}=\mathrm{Li}^{+}, \mathrm{Na}^{+}, \mathrm{K}^{+}\right)$ composite materials. The obtained Pt NPs are characterized extensively by a range of physical measurements including X-ray powder diffractions (PXRD), thermogravimetric analyses (TGA), inductively coupled plasma emission spectrometer (ICP), X-ray photoelectron spectroscopy (XPS) and transmission electron microscope (TEM). The morphology and size of Pt NPs can be easily tuned through changing the original supramolecular structures. The different interaction between $\left[\mathrm{PtCl}_{6}\right]^{2-}$ and $\mathrm{Me}_{10} \mathrm{CB}[5]$ capsulates affects the morphology and size of Pt NPs greatly. Such differences in geometric structures of Pt NPs have a significant impact on their catalytic performance. Moreover, the unique confinement effect provided by the supramolecular assembly as well as the special steric effect offered by macrocyclic $\mathrm{Me}_{10} \mathrm{CB}[5]$ suppresses the growth of Pt NPs during the reduction process. In addition, the atomic level uniform dispersion of Pt ions in ordered crystalline structure of the supramolecular assembly assures the uniform distribution of the final Pt NPs on the intact $\mathrm{Me}_{10} \mathrm{CB}$ [5], which acts as both a stabilizer and support. The Pt NPs obtained from $\mathrm{Na}^{+}$and $\mathrm{K}^{+}$constructed supramolecular assemblies exhibit high activity and chemoselectivity in catalytic hydrogenation of substituted nitrobenzenes to corresponding anilines under mild conditions. Meanwhile, the final Pt NPs also show an excellent stability in recycle test without dramatic loss of activity. This work demonstrates a novel, high-performance catalyst for the chemoselective hydrogenation reaction, and opens up a new approach for the preparation of nanocatalysts, which can be used in many other important fields, such as electrochemistry, energy science and environmental protection.
\end{abstract}

Keywords supramolecular crystalline materials; Pt nanoparticles; nitroarenes; catalytic hydrogenation

* E-mail: hongfangli@fjirsm.ac.cn

Received September 24, 2020; published October 26, 2020.

Supporting information for this article is available free of charge via the Internet at http://sioc-journal.cn.

Project supported by the National Natural Science Foundation of China (NSFC) (No. 21520102001) and Key Research Program of Frontier Science, Chinese Academy of Sciences (No. QYZDJ-SSW-SLH045).

项目受国家自然科学基金(No. 21520102001)和中国科学院前沿科学重点项目(No. QYZDJ-SSW-SLH045)资助. 


\section{1 引言}

苯胺及其衍生物是一类重要的化工中间体, 可用于 制备染料、农药、医药及聚合物等一系列化合物. 由于 苯胺类化合物的巨大作用, 开发廉价、高效的苯胺制备 方法依然是有机合成和化工工业中一个重要的研究方 向 ${ }^{[1-8]}$. 硝基苯直接催化加氢制备苯胺无疑是一种最为 直接、原子经济及环境友好的合成方法 ${ }^{[9-17]}$. 在催化加 氢反应中, 催化剂的催化活性对反应速率和产物质量具 有重要的影响. 当硝基苯分子含有多个可还原基团时, 对于不同基团的选择性催化加氢还原也是一个巨大的 挑战 ${ }^{[18-23]}$. 因而, 探索具有高选择性和高稳定性的加氢 催化剂具有重要的意义.

金属纳米粒子由于其独特的电子、磁性和光学性质, 在硝基芳烃还原反应中引起了广泛的关注 ${ }^{[24-26]}$. 以贵金 属为活性中心的 Pt、Pd、Rh 催化剂催化活性高、抗毒 能力强且稳定性好, 在实际生产中具有良好的便利性和 经济效应. 特别是超细贵金属纳米粒子具有较大的比表 面积, 更多的扭角位和边缘活性位点, 有利于催化加氢 反应的进行 ${ }^{[27-28]}$. 但是, 超细贵金属纳米晶由于较高的 比表面能, 反应过程中容易团聚, 造成催化剂失活 ${ }^{[29]}$. 因而, 贵金属纳米粒子通常负载在各类载体上, 利用载 体与贵金属纳米粒子间的相互作用来稳定贵金属纳米 粒子, 减弱纳米颗粒团聚, 提高催化剂的稳定性 ${ }^{[30]}$. 然 而, 选用一般的贵金属前驱体, 很难获得粒径小、形状 规则的纳米颗粒. 此外, 贵金属纳米粒子制备过程中常 用的还原剂如硼氢化钠、乙二醇等由于具有较强的还原 能力, 会导致纳米晶快速生长, 因而合成过程中通常需 要加入表面活性剂如柠檬酸钠、聚乙烯吡咯烷酮来控制 纳米粒子的粒径, 然而这些表面活性剂会在一定程度上 堵塞活性位点 ${ }^{[31]}$. 因而, 开发一种通用的、温和的合成 方法来制备负载型的超细贵金属纳米粒子催化剂仍是 当前的研究热点之一.

瓜环或称葫芦脲是苷腿通过亚甲基桥连形成的一 类新型大环分子化合物. 瓜环由于其特有的结构特性和 广阔的应用前景, 越来越受到主客体化学、自组装超分 子化学、环境化学、催化、材料化学、生物化学等国内 外相关学科研究者的广泛关注 ${ }^{[32-33]}$. 在催化领域, 瓜环 两端分布高极性的羰基基团对电荷稠密的金属阳离子 表现出高度的亲和力, 因而科研工作者们利用瓜环作为 载体成功制备了各种贵金属纳米粒子 ${ }^{[34-39]}$. 在前期的工 作中, 本课题组已成功合成了负载在瓜环上的各种贵金 属纳米颗粒 $(\mathrm{Pd}, \mathrm{Pt}, \mathrm{Au}, \mathrm{Ir}, \mathrm{Ru})$, 它们具有良好的催化活 性、稳定性和可重复使用性 ${ }^{[00-46]}$, 但贵金属金属纳米颗 粒的形貌和粒径上的精细控制还有待于提高. 因此, 本 工作改进合成方法，利用十甲基五元瓜环 $\left(\mathrm{Me}_{10} \mathrm{CB}[5]\right)$ 和氯铂酸根离子 $\left(\left[\mathrm{PtCl}_{6}\right]^{2-}\right)$ 在不同的碱金属离子 $\left(\mathrm{Li}^{+}\right.$, $\mathrm{Na}^{+}, \mathrm{K}^{+}$) 存在下自组装生成一系列超分子晶体材料. 继 而利用氢气原位还原晶体材料, 成功制备了尺寸约为
$3.0 \mathrm{~nm}$ 的超细 $\mathrm{Pt}$ 纳米粒子. 该 $\mathrm{Pt}$ 纳米粒子对硝基苯的 加氢反应具有优良的催化活性、化学选择性和稳定性.

\section{2 结果与讨论}

\section{1 催化剂的物理表征}

超细 Pt 纳米颗粒的合成过程见图 1 所示. 首先, 参 照前人的合成方法制备和分离 $\mathrm{Me}_{10} \mathrm{CB}[5]^{[47]}$. 然后, $\mathrm{Me}_{10} \mathrm{CB}[5]$ 和 $\left[\mathrm{PtCl}_{6}\right]^{2-}$ 阴离子分别放置在 $\mathrm{H}$ 管两侧，通过 溶液扩散法生长超分子晶体材料 ${ }^{[48]}$. 晶体材料在氢气 中原位还原生成超细 $\mathrm{Pt}$ 纳米粒子. $\mathrm{Me}_{10} \mathrm{CB}[5]$ 和 $\left[\mathrm{PtCl}_{6}\right]^{2-}$ 阴离子在不同碱金属离子 $\left(\mathrm{Li}^{+}, \mathrm{Na}^{+}, \mathrm{K}^{+}\right)$存在下，自组装 生成具有两类不同结构的一维链状超分子晶体. $\mathrm{Me}_{10} \mathrm{CB}[5]$ 端口的 5 个羰基 $\mathrm{O}$ 具有强电负性, 易于同溶 液中的碱金属阳离子配位. 由于离子半径较小, $\mathrm{Li}^{+}$与 $\mathrm{Me}_{10} \mathrm{CB}[5]$ 形成半封闭的分子胶囊 . 相邻的 $\left\{\mathrm{Li}_{2} \mathrm{Me}_{10} \mathrm{CB}[5]\right\}$ 分子胶囊通过在 $\mathrm{Li}^{+}$中心上的游离水配 体和端口羰基氧原子之间形成的氢键相互连接, 形成一 维超分子链结构. $\left[\mathrm{PtCl}_{6}\right]^{2-}$ 阴离子通过氢键作用与 $\left\{\mathrm{Li}_{2} \mathrm{Me}_{10} \mathrm{CB}[5]\right\}$ 分子胶囊自组装成超分子晶体(图 2a). 随着离子半径增大, $\mathrm{Na}^{+} 、 \mathrm{~K}^{+}$与 $\mathrm{Me}_{10} \mathrm{CB}[5]$ 端口的 5 个氧 配位, 形成全封闭的分子胶囊. 相邻的分子胶囊, 通过 碱金属离子与 $\left[\mathrm{PtCl}_{6}\right]^{2-}$ 中的 $\mathrm{Cl}^{-}$离子之间的离子键作用, 形成了两个同构的一维链状超分子晶体材料(图 2b). 合 成的超分子晶体材料在 $\mathrm{H}_{2}$ 氛围下加热还原, 生成负载 了超细 Pt 纳米颗粒的复合材料. 在这两类链状晶体材 料中, $\left[\mathrm{PtCl}_{6}\right]^{2-}$ 阴离子与瓜环分子胶囊间存在的不同相 互作用, 对原位还原后得到的超细 Pt 纳米颗粒的形貌 和粒径大小具有重要的影响.
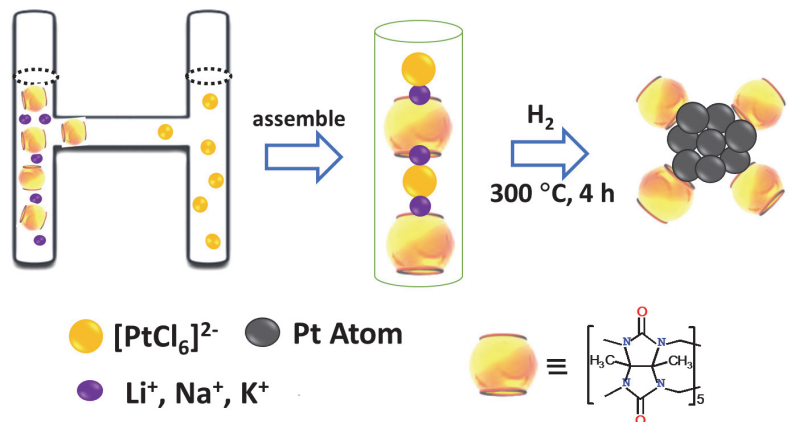

图 1 超细 $\mathrm{Pt}$ 纳米颗粒制备流程图

Figure 1 Schematic illustration of the fabrication of Pt nanoparticles

合成得到的超分子晶体材料在 $300{ }^{\circ} \mathrm{C}$ 氢气中还原 4 $\mathrm{h}$ 后, $\left[\mathrm{PtCl}_{6}\right]^{2-}$ 阴离子被原位还原生成超细 $\mathrm{Pt}$ 纳米颗粒. 从复合材料的 X-ray 粉末衍射(XRD)图谱(图 3)可以看 出, 加热还原后超分子晶体结构在一定程度上保持不 变, 而 XRD 图上未出现明显的 Pt 纳米颗粒的特征衍射 峰. 这主要是由于加热还原过程中, 部分超分子晶体骨 架未被还原, 而超分子晶体材料的高衍射强度, 掩盖了 超细 Pt 纳米颗粒的衍射峰(图 S1). 


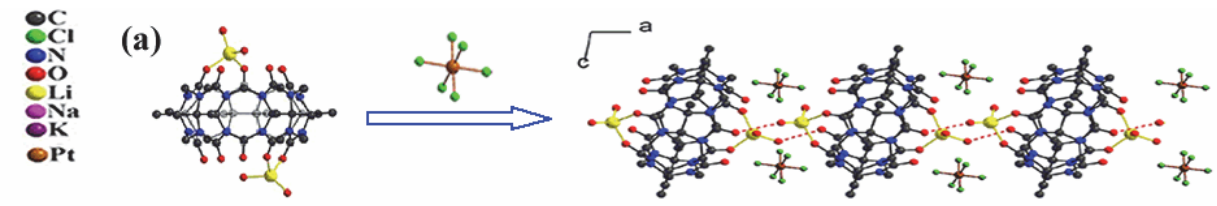

(b)

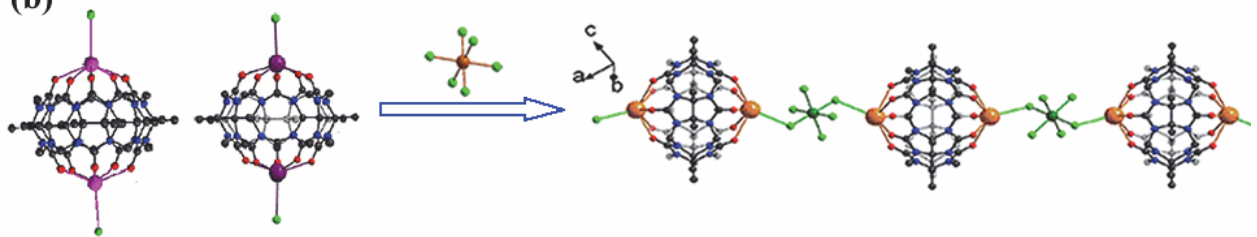

图 2 两种不同结构的一维链状超分子晶体

Figure 2 One-dimensional chain subunit of supramolecular crystallines

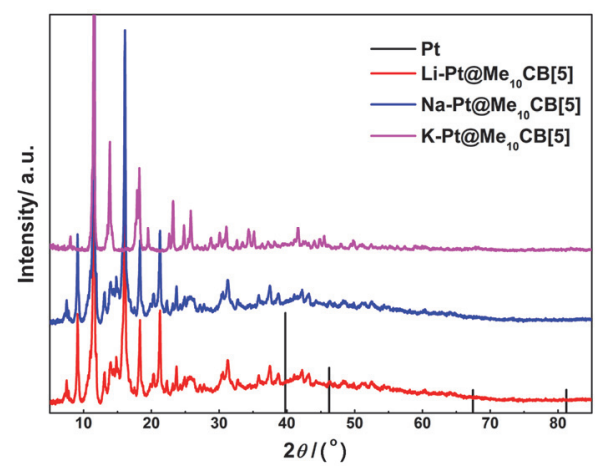

图 $3 \mathrm{M}-\mathrm{Pt} @ \mathrm{Me}_{10} \mathrm{CB}[5]$ 复合材料的 XRD 图

Figure 3 The XRD patterns of the M-Pt@ $\mathrm{Me}_{10} \mathrm{CB}[5]$ composites

通过 TEM 观察, 可以清晰看到还原生成的超细 Pt 纳米颗粒均匀分布在瓜环载体上. 复合材料 $\mathrm{Li}-\mathrm{Pt} @ \mathrm{Me}_{10} \mathrm{CB}[5]$ 中, Pt 纳米颗粒呈现不规则的形状, 粒 径大小不均一, 其平均粒径大小在 $4.4 \mathrm{~nm}$ (图 4a). 而 $\mathrm{Na}-\mathrm{Pt} @ \mathrm{Me}_{10} \mathrm{CB}[5]$ 和 K-Pt@ $\mathrm{Me}_{10} \mathrm{CB}[5]$ 中, 粒径均一的 球形 $\mathrm{Pt}$ 纳米颗粒均匀负载在瓜环上, 其平均粒径分别 为 $2.9 \mathrm{~nm}$ 和 $3.2 \mathrm{~nm}$ (图 $4 \mathrm{~b}$ 和 $4 \mathrm{c}$ ). 高分辨率 TEM 图上可 以看出, 合成得到的 $\mathrm{Pt}$ 纳米颗粒具有高结晶度, 晶面 间距为 $0.227 \mathrm{~nm}$ 和 $0.196 \mathrm{~nm}$, 分别对应于 $\mathrm{Pt}$ 的 $\{111\}$ 和 $\{200\}$ 晶面. 选区电子衍射(SAED)图呈现多晶衍射环, 分别属于面心立方 (fcc)Pt 纳米颗粒的 $\{111\} 、\{200\}$ 、 $\{220\} 、\{311\}$ 晶面(图 4d). 在超分子晶体材料中, Pt 呈现 原子级别均匀分布, 因而原位还原后, Pt 纳米粒子的分 布比较均一. 还原过程中, $\mathrm{Me}_{10} \mathrm{CB}[5]$ 的大环空间效应有 效抑制了 Pt 纳米粒子的团聚, 确保超细 Pt 纳米颗粒的 生成. $\mathrm{Li}^{+}$构筑的一维超分子晶体中, $\left[\mathrm{PtCl}_{6}\right]^{2-}$ 与瓜环分 子胶囊通过比较弱的氢键作用连接在一起, 因而还原过 程中, 瓜环分子对合成的 Pt 纳米颗粒的限域效应比较 小, 因而得到 Pt 纳米粒子粒径较大, 尺寸不均一. 而 $\mathrm{Na}^{+}$和 $\mathrm{K}^{+}$构筑的两个同构的晶体材料中, $\left[\mathrm{PtCl}_{6}\right]^{2-}$ 通过 碱金属- $\mathrm{Cl}$ 之间的离子键作用连接在一起, 每个 $\left[\mathrm{PtCl}_{6}\right]^{2-}$ 周边连接 6 个瓜环分子胶囊, 有效防止 $\mathrm{Pt}$ 纳米颗粒的团 聚. 而 $\mathrm{Na}^{+}$和 $\mathrm{K}^{+}$两个同构晶体还原后得到的 $\mathrm{Pt}$ 纳米颗
粒, 粒径大小存在微小的差异, 这主要是由于不同碱金 属调节了 $\mathrm{Pt}$ 中心的电子密度, 进而影响了羰基 O-Pt 之 间的相互作用. Pt 纳米颗粒形貌和粒径大小的差异, 对 其催化性能具有重要的影响.
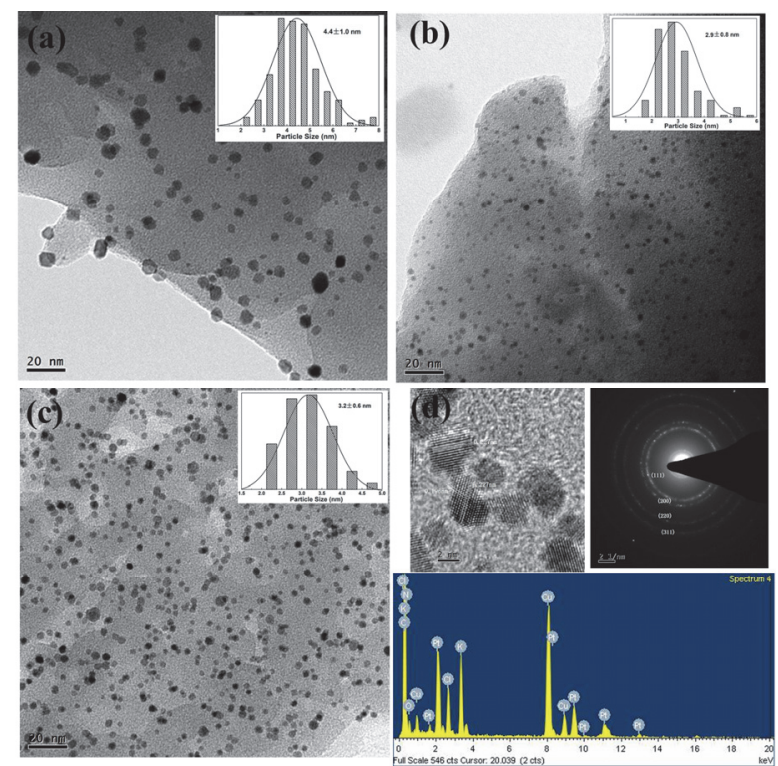

图 4 (a) Li-Pt@ $\mathrm{Me}_{10} \mathrm{CB}[5]$, (b) $\mathrm{Na}-\mathrm{Pt} @ \mathrm{Me}_{10} \mathrm{CB}[5]$ 和 (c) $\mathrm{K}-\mathrm{Pt} @$ $\mathrm{Me}_{10} \mathrm{CB}[5]$ 的透射电镜图, 相应内插图为 $\mathrm{Pt}$ 纳米颗粒的粒径分布图. (d) $\mathrm{K}-\mathrm{Pt} @ \mathrm{Me}_{10} \mathrm{CB}[5]$ 的能谱以及高分辨透射电镜和选区电子衍射图.

Figure 4 The TEM images of (a) Li-Pt@ $\mathrm{Me}_{10} \mathrm{CB}[5]$, (b) $\mathrm{Na}-\mathrm{Pt} @$ $\mathrm{Me}_{10} \mathrm{CB}[5]$ and (c) K-Pt@ $\mathrm{Me}_{10} \mathrm{CB}[5]$. The inset are the corresponding diameter distribution histogram of the Pt NPs. (d) EDS analysis, high-resolution TEM image and the SAED pattern of the Pt NPs denoted in (c)

采用 X射线光电子能谱(XPS)进一步分析超细 $\mathrm{Pt}$ 纳 米颗粒的价态. 通过对 Pt $4 \mathrm{f}$ 精细谱分析，体系中包括金 属 $\mathrm{Pt}^{0}$ 、氧化态 $\mathrm{Pt}^{2+}$ 和 $\mathrm{Pt}^{4+}$. 如图 5 所示, $71.9 \mathrm{eV}$ 和 75.4 $\mathrm{eV}$ 的一对峰属于 $\mathrm{Pt}^{0}$ 的 $4 \mathrm{f} 7 / 2$ 和 $4 \mathrm{f} 5 / 2 ; 76.5 \mathrm{eV}$ 和 73.1 $\mathrm{eV}$ 的峰属于氧化态 $\mathrm{Pt}^{2+}$; 而 $77.8 \mathrm{eV}$ 和 $74.4 \mathrm{eV}$ 的属于 晶体中尚未还原的 $\mathrm{Pt}^{4+}$. 这三个价态的 $\mathrm{Pt}$ 含量分别为 $45.6 \%, 14.2 \%$ 和 $40.2 \%$. 因而, 晶体材料中近一半的 $\mathrm{Pt}^{4+}$ 被原位还原生成超细 $\mathrm{Pt}$ 纳米颗粒, 零价 $\mathrm{Pt}$ 纳米颗粒具 有优良的还原硝基苯生成相应苯胺的催化活性. 


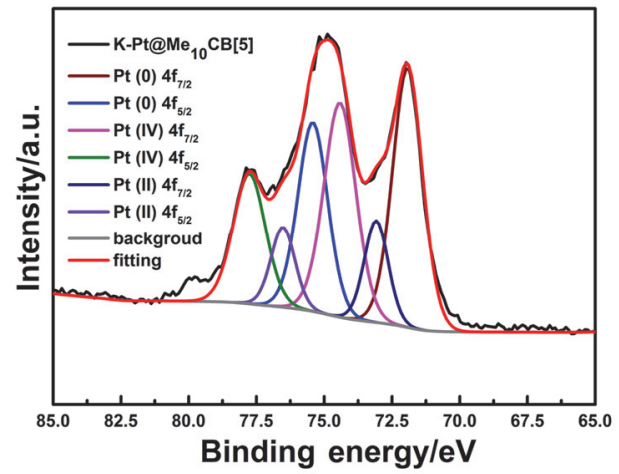

图 $5 \mathrm{~K}-\mathrm{Pt} @ \mathrm{Me}_{10} \mathrm{CB}[5]$ 中 $\mathrm{Pt} 4 \mathrm{f}$ 光电子能谱图

Figure 5 XPS spectra of Pt $4 \mathrm{f}$ in $\mathrm{K}-\mathrm{Pt} @ \mathrm{Me}_{10} \mathrm{CB}[5]$

\section{2 硝基苯加氢催化性能表征}

为了探索催化剂的催化性能, 将合成的 Pt 催化剂 应用于硝基苯加氢反应. 如表 1 所示, 所有反应均在常 温常压下乙醇溶液中进行. 首先从催化剂的用量和反应 时间来优化反应条件. 未加入 $\mathrm{Pt}$ 催化剂, 反应几乎不能 进行, 只产生痕量的苯胺(表 1, Entry 1). 随着加入 Pt 催 化剂量的逐渐增加, 苯胺含量逐渐提高, 加入 $0.5 \mathrm{~mol} \%$ $\mathrm{Pt}$ 催化剂后, 反应 $1 \mathrm{~h}$, 苯胺产率达到 $65.7 \%$ (表 1 , Entries $2 \sim 6$ ). 固定催化剂加入量为 $0.5 \mathrm{~mol} \%$, 反应时间 从 $1.0 \mathrm{~h}$ 延长到 $1.5 \mathrm{~h}$, 苯胺产率高达 $98.2 \%$ (表 1, Entry 7). 因此, 我们篮选出催化剂用量为 $0.5 \mathrm{~mol} \%$, 反应时 间 $1.5 \mathrm{~h}$ 为最佳反应条件.

表 1 超细 Pt 纳米颗粒催化加氢硝基苯

Table 1 Hydrogenation of nitrobenzene to aniline catalyzed by ultra-small Pt nanoparticles

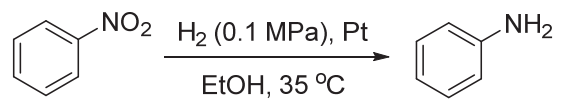

\begin{tabular}{cccccc}
\hline Entry & Catalyst & $\begin{array}{c}\mathrm{Pt} \\
(\mathrm{mol} \%)\end{array}$ & Solvent & Time/h & Yield/\% \\
\hline 1 & Blank & 0 & Ethanol & 1.0 & Trace \\
2 & $\mathrm{~K}-\mathrm{Pt} @ \mathrm{Me}_{10} \mathrm{CB}[5]$ & 0.05 & Ethanol & 1.0 & 2.04 \\
3 & $\mathrm{~K}-\mathrm{Pt} @ \mathrm{Me}_{10} \mathrm{CB}[5]$ & 0.1 & Ethanol & 1.0 & 4.4 \\
4 & $\mathrm{~K}-\mathrm{Pt} @ \mathrm{Me}_{10} \mathrm{CB}[5]$ & 0.3 & Ethanol & 1.0 & 31.5 \\
5 & $\mathrm{~K}-\mathrm{Pt} @ \mathrm{Me}_{10} \mathrm{CB}[5]$ & 0.5 & Ethanol & 1.0 & 65.7 \\
6 & $\mathrm{~K}-\mathrm{Pt} @ \mathrm{Me}_{10} \mathrm{CB}[5]$ & 1.0 & Ethanol & 1.0 & 100 \\
7 & $\mathrm{~K}-\mathrm{Pt} @ \mathrm{Me}_{10} \mathrm{CB}[5]$ & 0.5 & Ethanol & 1.5 & 98.2 \\
8 & $\mathrm{~K}-\mathrm{Pt} @ \mathrm{Me}_{10} \mathrm{CB}[5]$ & 0.5 & Ethanol & 2.0 & 100 \\
9 & $\mathrm{Li}-\mathrm{Pt} @ \mathrm{Me}_{10} \mathrm{CB}[5]$ & 0.5 & Ethanol & 1.5 & 74.9 \\
10 & $\mathrm{Na}-\mathrm{Pt} @ \mathrm{Me}_{10} \mathrm{CB}[5]$ & 0.5 & Ethanol & 1.5 & 98.9 \\
\hline
\end{tabular}

在最优反应条件下, 对合成的三个不同 Pt 催化剂 进行催化性能比较 (表 1, Entries 7, 9, 10), 其中 $\mathrm{K}-\mathrm{Pt} @ \mathrm{Me}_{10} \mathrm{CB}[5]$ 和 $\mathrm{Na}-\mathrm{Pt} @ \mathrm{Me}_{10} \mathrm{CB}[5]$ 催化剂苯胺产率 均接近 99\%, 而 Li-Pt@ $\mathrm{Me}_{10} \mathrm{CB}[5]$ 催化剂的产率只有 $74.9 \%$. 三者催化活性的差异是由 $\mathrm{Pt}$ 纳米粒子的粒径大 小和形貌不同引起的. 众所周知, 随着粒子尺寸的减小,
$P t$ 纳米晶具有更多的扭角位、边缘位活性位点，有利于 提高 $\mathrm{Pt}$ 催化剂的催化效率和催化选择性 ${ }^{[28]}$. 因而 $\mathrm{Na}-\mathrm{Pt} @ \mathrm{Me}_{10} \mathrm{CB}[5]$ 和 K-Pt@ $\mathrm{Me}_{10} \mathrm{CB}[5]$ 催化剂中, 形貌 规则, 粒径均匀的超细 $\mathrm{Pt}$ 纳米粒子 (约 $3.0 \mathrm{~nm}$ ) 比 $\mathrm{Li}-\mathrm{Pt}-\mathrm{Me}_{10} \mathrm{CB}[5]$ 中较大的 Pt 纳米颗粒具有更加优良的 催化活性. 此外, Na-Pt@ $@ \mathrm{Me}_{10} \mathrm{CB}[5]$ 和 K-Pt@ $@ \mathrm{Me}_{10} \mathrm{CB}[5]$ 这两个材料中，瓜环端口羰基的电子云偏向与羰基 $\mathrm{O}$ 直 接相连的 Pt 原子, 从而改变了 $\mathrm{Pt}$ 原子的表面电荷密度, 进而影响其催化活性 ${ }^{[45]}$.

为了实现工业化应用，催化剂不仅要有高活性、高 选择性, 还需要高稳定性. 本工作中, 测试了 3 个 $\mathrm{Pt}$ 催 化剂的 5 次循环催化活性. 从图 6a 可以看出, $\mathrm{Na}-\mathrm{Pt} @ \mathrm{Me}_{10} \mathrm{CB}[5]$ 和 K-Pt@ $\mathrm{Me}_{10} \mathrm{CB}[5]$ 这两个催化剂经 过 5 次循环使用后, 产率只有轻微下降, 可能是由于循 环过程中催化剂不可避免流失引起. 这两个催化剂中 $\mathrm{Pt}$ 与瓜环端口羰基 $\mathrm{O}$ 之间强的相互作用稳定了超细 $\mathrm{Pt}$ 纳 米粒子, 保证其高稳定性 ${ }^{[45]}$. 而 $\mathrm{Li}-\mathrm{Pt} @ \mathrm{Me}_{10} \mathrm{CB}[5]$ 中由 于相互作用比较弱, 因而循环使用 5 次后, 产率急剧下 降. 反应后催化剂的 TEM 图片可以看出, Pt 纳米颗粒保 持其均一的形貌, 没有团聚生成 Pt 大颗粒(图 6b). 催化 反应后, 反应溶剂进行 ICP 测试, Pt 的含量可以忽略不 计，因此合成的 Pt 催化剂具有优异的稳定性.
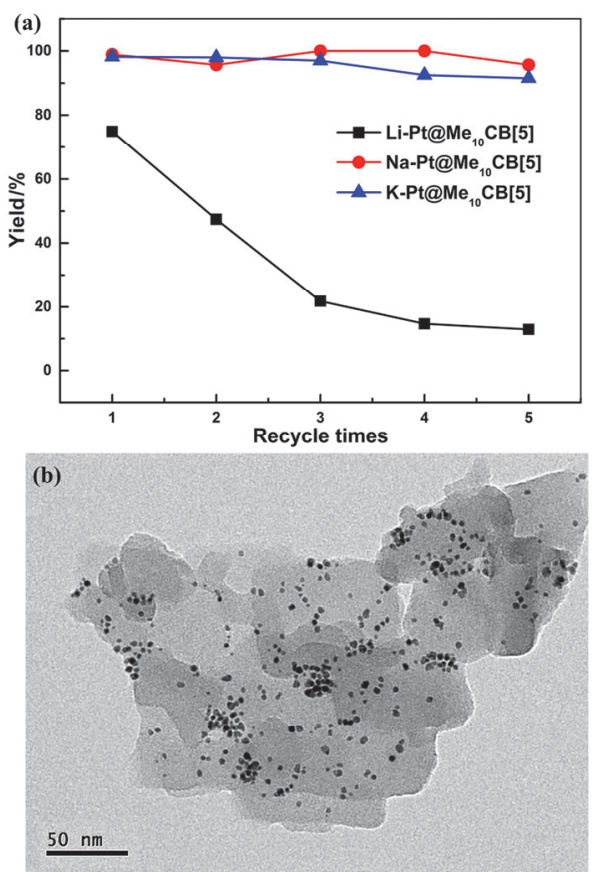

图 6 (a) 催化剂循环稳定性测试和(b) 5 次循环使用后 Pt 纳米颗粒的 TEM 图

Figure 6 (a) The reusability of catalysts in hydrogenation of nitrobenzene and (b) TEM image for K-Pt@ $\mathrm{Me}_{10} \mathrm{CB}[5]$ after fifth recycles catalysis

根 据 优 化后的反应条 件, 将 催 化 剂 $\mathrm{K}-\mathrm{Pt} @ \mathrm{Me}_{10} \mathrm{CB}[5]$ 进一步应用于催化加氢其他硝基苯衍 生物, 拓展其反应底物的范围. K-Pt@ $\mathrm{Me}_{10} \mathrm{CB}[5]$ 催化剂 
成功将带有不同供/给电子取代基(对羟基、对甲基、对 氯基、对醛基等)的硝基苯衍生物转换成相应的苯胺衍 生物, 获得了很高的产率(表 2, Entries 2 7). 从表格中 的数据可以看出, 给电子基团的存在能一定程度提高硝 基苯加氢反应效率, 在较短时间内其产率就可以达到 99\%以上(表 2, Entry 3). 当硝基苯衍生物分子中存在其 他可还原基团时, 催化剂的高选择性还原显得尤为重 要. 含有酯基的硝基苯衍生物在还原过程中, 酯基保持 不变, 其转化率和选择性均高于 $99 \%$ (表 2, Entry 4). 当 分子中存在易还原的羰基官能团(如酮和醛), 硝基也能 被高选择性地还原成氨基(表 2, Entries 5,6), 选择性分 别是 $99 \%$ 和 $96 \%$. 氯代硝基苯催化加氢制备氯代苯胺是 一个复杂的反应过程, 催化加氢过程中会出现大量的副 反应, 其中加氢脱氯反应是最严重的副反应. 而 $\mathrm{K}-\mathrm{Pt} @ \mathrm{Me}_{10} \mathrm{CB}[5]$ 催化剂有效促进对氯硝基苯的加氢反 应, 产物选择性高达 $85 \%$, 其中脱氯产物苯胺占 $15 \%$ (表 2, Entry 7). 上述研究结果表明, 合成得到的 Pt 催化剂 是一个高效、高选择性的催化剂, 它可以将带有不同官 能团的硝基苯衍生物转化为相应的苯胺衍生物.

表 $2 \mathrm{~K}-\mathrm{Pt} @ \mathrm{Me}_{10} \mathrm{CB}[5]$ 催化不同的芳香硝基化合物

Table 2 Reduction of various nitroarenes catalyzed by $\mathrm{K}-\mathrm{Pt} @ \mathrm{Me}_{10^{-}}$ $\mathrm{CB}[5]^{a}$

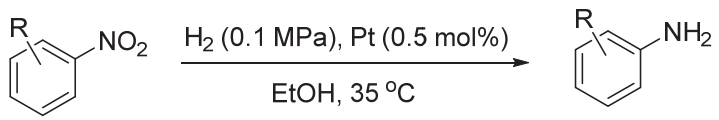

\section{3 结论}

通过原位还原瓜环和 $\mathrm{Pt}$ 构筑的一系列超分子晶体 材料制备了粒径为 $3.0 \mathrm{~nm}$ 的超细 $\mathrm{Pt}$ 纳米颗粒. 该 Pt 纳 米颗粒在硝基苯衍生物加氢还原反应中表现出高效的 催化活性、优异的化学选择性和高稳定性. 该合成方法 的突出优势体现在如下两个方面: (1) Pt 纳米颗粒的大 小和形貌可以通过调节原始晶体结构得到有效控制; (2) $\mathrm{Me}_{10} \mathrm{CB}[5]$ 的空间限域效应有限抑制 $\mathrm{Pt}$ 纳米颗粒团聚, 而瓜环端口羰基 $\mathrm{O}$ 同 $\mathrm{Pt}$ 原子间的相互作用，提高了 $\mathrm{Pt}$ 纳米粒子的稳定性; (3) 合成过程中无需添加额外的表 面活性剂来稳定 $\mathrm{Pt}$ 纳米粒子, 避免表面活性剂堵塞催 化剂的活性位点. 该合成方法还可以进一步拓展, 将其 它贵金属 $(\mathrm{Pd}, \mathrm{Au}, \mathrm{Ag}, \mathrm{Rh})$ 纳米粒子负载到瓜环载体上, 从而为负载型纳米催化剂的合成提供了一个新的思路.

\section{4 实验部分}

\section{1 试剂}

各种化合物, 如氯化锂 $(\mathrm{LiCl}) 、$ 氯化钠 $(\mathrm{NaCl})$ 、氯化 钾 $(\mathrm{KCl}) 、$ 六水合氯铂酸 $\left(\mathrm{H}_{2} \mathrm{PtCl}_{6} \cdot 6 \mathrm{H}_{2} \mathrm{O}\right)$ 等都是市售分析 纯试剂, 未经纯化直接使用. $\mathrm{Me}_{10} \mathrm{CB}$ [5]按照参考文献制 备和分离 ${ }^{[47]}$. 实验中使用的超纯水(18 M $\Omega$ )由 Millipore 超纯水机提供.

\section{2 实验仪器和测试条件}

样品的物相和纯度采用 X-ray 粉末衍射仪(XRD)进 行检测. 仪器型号: Rigaku DMAX 2500 衍射仪, X-ray 射线源为 $\mathrm{Cu} / \mathrm{K} \alpha(\lambda=0.154178 \mathrm{~nm})$. 样品的形貌采用透 射电子显微镜(FEI TECNAI G2 F20)进行观察, 加速电 压为 $200 \mathrm{kV}$. 测试前, 首先将样品超声分散在乙醇中, 然后将悬浊液滴到涂有碳膜的铜网上，空气中干燥备 用. 贵金属 $\mathrm{Pt}$ 含量用电感耦合等离子发射光谱仪 (ICP-AES)测得, 仪器型号为 Jobin Yvon Ultima 2. X 射 线光电子能谱(XPS)分析使用 ESCALAB 250 Xi XPS system 型能谱仪在真空状态下测试.

\section{$4.3 \mathrm{M}-\mathrm{Pt} @ \mathrm{Me}_{10} \mathrm{CB}[5]$ 复合材料的合成}

首先根据课题组之前报道的合成方法 ${ }^{[48]}$, 在不同 碱金属离子 $\left(\mathrm{Li}^{+}, \mathrm{Na}^{+}, \mathrm{K}^{+}\right)$存在下, $\mathrm{Me}_{10} \mathrm{CB}[5]$ 和 $\left[\mathrm{PtCl}_{6}\right]^{2-}$ 阴离子自组装形成两种不同结构的一维链状超分子晶 体. 合成的超分子晶体经过洗涤、常温晾干后, 转入管 式炉中, $\mathrm{H}_{2}$ 气氛下在 $300{ }^{\circ} \mathrm{C}$ 加热还原 $4 \mathrm{~h}$, 得到一系列 负载 Pt 超细纳米颗粒的 $\mathrm{M}-\mathrm{Pt} @ \mathrm{Me}_{10} \mathrm{CB}[5](\mathrm{M}=\mathrm{Li}, \mathrm{Na}$, $\mathrm{K})$ 的复合材料. 得到的复合材料水洗除去残留的碱金属 阳离子和氯离子, 干燥后备用.

\section{4 催化性能研究}

硝基苯的加氢反应在室温常压下进行. 将 $1.0 \mathrm{mmol}$ 硝基芳烃、定量的 $\mathrm{Pt}$ 催化剂和 $3.0 \mathrm{~mL}$ 乙醇加入 $20 \mathrm{~mL}$ 史莱克瓶. 反应体系先抽真空后通入氢气，重复操作三 
次除去反应体系中的空气. 反应物在 $\mathrm{H}_{2}$ 气氛下, 以每 分钟 600 转的速度搅拌反应一定时间. 反应结束后, 混 合物转移到离心管中, 离心分离出固体催化剂. 采用气 相色谱(430 GC)对液相进行分析, 测定加氢反应的转化 率和产物的选择性. 为了考察催化剂的循环催化活性, 反应过一次的催化剂离心分离、乙醇清洗后, 重新加入 新的催化底物, 按照上述的催化条件, 进行下一轮的催 化反应.

\section{References}

[1] Blaser, H.-U.; Steiner, H.; Studer, M. ChemCatChem 2009, 1, 210.

[2] Lara, P.; Philippot, K. Catal. Sci. Technol. 2014, 4, 2445.

[3] Vile, G.; Albani, D.; Almora-Barrios, N.; Lopez, N.; Perez-Ramírez, J. Chem CatChem 2016, 8, 21.

[4] Song, J.; Huang, Z.; Pan, L.; Li, K.; Zhang, X.; Wang, L.; Zou, J. Appl. Catal., B 2018, 227, 386.

[5] Kadam, H. K.; Tilve, S. G. RSC Adv. 2015, 5, 83391.

[6] Zhang, L.; Zhou, M.; Wang, A.; Zhang, T. Chem. Rev. 2020, 120, 683.

[7] Li, X.; Wang, Z.; Mao, S.; Chen, Y.; Tang, M.; Li, H.; Wang, Y. Chin. J. Chem. 2018, 36, 1191.

[8] Li, S.; Wang, F.; Liu, Y.; Cao, Y. Chin. J. Chem. 2017, 35, 591.

[9] Westerhaus, F. A.; Jagadeesh, R. V.; Wienhofer, G.; Pohl, M M.; Radnik, J.; Surkus, A. E.; Rabeah, J.; Junge, K.; Junge, H.; Nielsen, M.; Bruckner, A.; Beller, M. Nat. Chem. 2013, 5, 537.

[10] Yang, N.; Cheng, H.; Liu, X.; Yun, Q.; Chen, Y.; Li, B.; Chen B.; Zhang, Z.; Chen, X.; Lu, Q.; Huang, J.; Huang, Y.; Zong, Y.; Yang, Y.; Gu, L.; Zhang, H. Adv. Mater. 2018, 30, 1803234.

[11] Yang, F.; Wang, M.; Liu, W.; Yang, B.; Wang, Y.; Luo, J.; Tang Y.; Hou, L.; Li, Y.; Li, Z.; Zhang, B.; Yang, W.; Li, Y. Green Chem. 2019, 21, 704

[12] Wei, Z.; Wang, J.; Mao, S.; Su, D.; Jin, H.; Wang, Y.; Xu, F.; Li, H.; Wang, Y. ACS Catal. 2015, 5, 4783 .

[13] Zhou, P.; Jiang, L.; Wang, F.; Deng, K.; Lv, K.; Zhang, Z. Sci. Adv. 2017, 3, e1601945.

[14] Sun, Q.; Wang, N.; Zhang, T.; Bai, R.; Mayoral, A.; Zhang, P.; Zhang, Q.; Terasaki, O.; Yu, J. Angew. Chem., Int. Ed. 2019, 58, 18570.

[15] Fukui, M.; Koshida, W.; Tanaka, A.; Hashimoto, K.; Kominami, H. Appl. Catal., B 2020, 268, 118446.

[16] Tomkins, P.; Gebauer-Henke, E.; Muller, T. E. ChemCatChem 2016, 8,546 .

[17] Tomkins, P.; Gebauer-Henke, E.; Leitner, W.; Muller, T. E. ACS Catal. 2015, 5, 203.

[18] Chen, G.; Xu, C.; Huang, X.; Ye, J.; Gu, L.; Li, G.; Tang, Z.; Wu, B.; Yang, H.; Zhao, Z.; Zhou, Z.; Fu, G.; Zheng, N. Nat. Mater. 2016, 15,564 .
[19] Qiao, B.; Wang, A.; Yang, X.; Allard, L. F.; Jiang, Z.; Cui, Y.; Liu, J.; Li, J.; Zhang, T. Nat. Chem. 2011, 3, 634.

[20] Wei, H.; Liu, X.; Wang, A.; Zhang, L.; Qiao, B.; Yang, X.; Huang, Y.; Miao, S.; Liu, J.; Zhang, T. Nat. Commun. 2014, 5, 5634.

[21] Tomkins, P.; Gebauer Henke, E.; Leitner, W.; Muller, T. E. ACS Catal. 2015, 5, 203.

[22] Yoshida, H.; Igarashi, N.; Fujita, S. i.; Panpranot, J.; Arai, M. Catal. Lett. 2015, 145, 606.

[23] Berguerand, C.; Yarulin, A.; Cardenas Lizana, F.; Warna, J.; Sulman, E.; Murzin, D. Y.; Kiwi Minsker, L. Ind. Eng. Chem. Res. 2015, 54, 8659.

[24] Liu, H. L.; Nosheen, F.; Wang, X. Chem. Soc. Rev. 2015, 44, 3056.

[25] Li, D. D.; Yu, S.-H.; Jiang, H.-L. Adv. Mater. 2018, 1705112.

[26] Hervés, P.; Pérez-Lorenzo, M.; Liz-Marzán, L. M.; Dzubiella, J.; Lu, Y.; Ballauff, M. Chem. Soc. Rev. 2012, 41, 5577.

[27] Jia, W.; Wu, Y.; Chen, Y. F.; He, D. S.; Li, J. P.; Wang, Y.; Wang, Z.; Zhu, W.; Chen, C.; Peng, Q.; Wang, D. S.; Li, Y. D. Nano Res. 2016, 9, 584.

[28] Mao, J. J.; Chen, Y. J.; Pei, J. J.; Wang, D. S.; Li, Y. D. Chem. Commun. 2016, 52, 5985.

[29] Yang, Q.; Xu, Q.; Jiang, H. L. Chem. Soc. Rev. 2017, 46, 4774.

[30] Zhao, S.; Tang, Z.; Guo, S.; Han, M.; Zhu, C.; Zhou, Y.; Bai, L.; Gao, J.; Huang, H.; Li, Y.; Liu, Y.; Kang, Z. ACS Catal. 2017, 8, 188

[31] Lei, S.; Li, Q. H.; Kang, Y.; Gu, Z. G.; Zhang, J. Appl. Catal., B 2019, $245,1$.

[32] Barrow, S. J.; Kasera, S.; Rowland, M. J.; Barrio, J.; Scherman, O. A. Chem. Rev. 2015, 115, 12320.

[33] Assaf, K. I.; Nau, W. M. Chem. Soc. Rev. 2015, 44, 394.

[34] Corma, A.; García, H.; Montes-Navajas, P.; Primo, A.; Calvino, J.; Trasobares, S. Chem. Eur. J. 2007, 13, 6359.

[35] Montes-Navajas, P.; Garcia, H. J. Phys. Chem. C 2010, 114, 18847.

[36] Taylor, R. W.; Lee, T. C.; Scherman, O. A.; Esteban, R.; Aizpupurua, J.; Huang, F. M.; Baumberg, J. J.; Mahajan, S. ACS Nano 2011, 5, 3878.

[37] Lee, T.-C.; Scherman, O. A. Chem. Eur. J. 2012, 18, 1628.

[38] Lu, X.; Masson, E. Langmuir 2011, 27, 3051.

[39] Premkumar, T.; Lee, Y.; Geckeler, K. E. Chem. Eur. J. 2010, 16, 11563.

[40] Cao, M.; Wu, D.; Gao, S.; Cao, R. Chem. Eur. J. 2012, 18, 12978.

[41] Cao, M.; Lin, J.; Yang, H.; Cao, R. Chem. Commun. 2010, 46, 5088.

[42] Cao, M.; Wei, Y.; Gao, S.; Cao, R. Catal. Sci. Technol. 2012, 2, 156.

[43] Li, H. F.; Lu, J.; Lin, J. X.; Cao, R. Inorg. Chem. 2014, 53, 5692.

[44] Chen, R.; Cao, M.; Yang, W.; Wang, H.; Zhang, S.; Li, H.; Cao, R.; Chem. Commun. 2019, 55, 9805.

[45] You, H.; Wu, D.; Chen, Z.; Sun, F.; Zhang, H.; Chen, Z.; Cao, M.; Zhuang, W.; Cao, R. ACS Energy Lett. 2019, 4, 1301.

[46] Gong, Z.; Wu, D.; Cao, M.; Zhao, C.; Cao, R. Chem. Commun. 2020, 56, 9392 .

[47] Shih, N.-Y. Diss. Abstr. Int., B 1982, B42, 4071.

[48] Chen, R.; Cao, M.; Wang, J.; Li, H.; Cao, R. Chem. Commun. 2019, 55, 11687.

(Cheng, B.) 